\title{
6

\section{Novel Ablation Therapy Using Endoscopic Irreversible Electroporation in the Bile Duct: A Pilot Animal Study}

\author{
Kang Won Lee ${ }^{1 *}$, Jae Min Lee ${ }^{1 *}$, Hyuk Soon Choi', Eun Sun Kim', Bora Keum ${ }^{1}$, Yeon Seok Seo', Yoon Tae Jeen', Soon Ho Um', Hong \\ Sik Lee', Hoon Jai Chun', Chang Duck Kim ${ }^{1}$, Chi Hyuk Oh${ }^{2}$ and Hong Bae Kim ${ }^{3}$
}

${ }^{1}$ Division of Gastroenterology and Hepatology, Department of Internal Medicine, Korea University College of Medicine, Seoul, ${ }^{2}$ Division of Gastroenterology and Hepatology, Department of Internal Medicine, Kyung Hee University College of Medicine, Seoul, ${ }^{3}$ Department of Biosystems and Biomaterials Science and Engineering, Seoul National University, Seoul, Korea

Background/Aims: Irreversible electroporation (IRE) is a relatively new ablation method. However, the application of IRE ablation in the treatment of biliary disease has not been attempted. A minimally invasive approach using endoscopic retrograde cholangiopancreatography (ERCP) can be a novel therapeutic modality for IRE ablation. In this study, we aimed to investigate the feasibility of endoscopic IRE for the biliary tract using an animal model.

Methods: A new catheter-type electrode was developed for endoscopic IRE ablation of the biliary tract. We performed ERCP and endoscopic IRE ablations in the normal common bile duct of Yorkshire pigs. The experimental setting of IRE was $500 \mathrm{~V} / \mathrm{cm}$ (50 pulses, 100- $\mu$ s length). The animals were sacrificed after $24 \mathrm{hr}$, and the ablated bile duct was examined.

Results: Well-demarcated focal color changes were observed on the mucosa of the common bile duct. The depth of change after IRE was confined to the mucosal and submucosal layers. Apoptotic changes in the bile duct were observed only around the IRE ablation area. Immunohistochemistry assay showed cell death in the bile duct along the electrode.

Conclusions: Endoscopic IRE ablation using ERCP was successfully performed in the common bile duct. It can be a potential option for the treatment of biliary tumors. Clin Endosc 2021;54:413-419

Key Words: Ablation; Bile duct; Endoscopic retrograde cholangiopancreatography; Irreversible electroporation

\section{INTRODUCTION}

Cholangiocarcinoma is among the types of cancer with a poor prognosis, and only a few patients with this diagnosis can undergo surgery with complete resection. ${ }^{1}$ Several chemotherapeutic drugs have been introduced; however, the poor prognosis and short survival period limit the treatment

\footnotetext{
Received: May 8, 2020 Revised: July 7, 2020

Accepted: July 9, 2020

Correspondence: Jae Min Lee

Division of Gastroenterology and Hepatology, Department of Internal Medicine,

Korea University College of Medicine, Korea University Medical Center, 73 Goryeodae-ro, Seongbuk-gu, Seoul 02841, Korea

Tel: +82-2-920-6555, Fax: +82-2-953-1943, E-mail: jmlee1202@gmail.com ORCID: https://orcid.org/0000-0001-9553-5101

*These authors contributed equally to this study.

(c) This is an Open Access article distributed under the terms of the Creative Commons Attribution Non-Commercial License (http://creativecommons.org/ licenses/by-nc/3.0) which permits unrestricted non-commercial use, distribution, and reproduction in any medium, provided the original work is properly cited.
}

of cholangiocarcinoma. ${ }^{2}$ Under these circumstances, several studies evaluating local treatments for cholangiocarcinoma in addition to systemic chemotherapy have been conducted. ${ }^{3}$ As a prime example of ablation therapy, irreversible electroporation (IRE) can destroy cancer cells in a nonthermal manner. ${ }^{4}$ Previous electrical treatments used radiofrequency applications; however, their clinical applications have been limited owing to the thermal effect. ${ }^{5}$ IRE can disrupt cellular homeostasis confined to the target lesion area, without damaging the surrounding vascular structures, thus inducing apoptosis of the parenchyma. ${ }^{6}$

IRE is based on the principle of electroporation. Electroporation is a technology that introduces electrical pulses with a width from $1 \mathrm{~ns}$ to $1 \mathrm{~ms}$ and an energy intensity from 0.4 to 1 $\mathrm{kV} / \mathrm{cm}$ to the cell, forming a nanometer-sized pore on the surface of the cell membrane. This technology has been applied to various biotechnology fields for decades since its first discovery in the 1970s. ${ }^{7}$ The micropore on the surface of a cell mem- 
brane produced under appropriate electrical pulse conditions has a reversible characteristic; that is, it disappears again after a period of time (reversible electroporation). On the contrary, a micropore produced under stronger electrical pulses remains open, which compromises the cellular homeostasis and eventually destroys the cell. This method is referred to as IRE. ${ }^{8}$

IRE was approved with restrictions by the Food and Drug Administration in 2011 after it was theoretically introduced in 2005. Considering the results of previous studies, IRE could be an effective and feasible treatment option for advanced cholangiocarcinoma. In fact, previous studies have reported that combining IRE and systemic chemotherapy showed good results in pancreatic cancer. ${ }^{9}$ Therefore, IRE is clinically applicable in solid organs such as the liver and pancreas. However, the IRE procedure has a threshold when inserting the IRE probe directly into the lesion with the patient's abdomen opened in the operating room. ${ }^{10}$ As open surgery under general anesthesia can cause a burden to patients with advanced cancer, IRE is not commonly performed in real clinical practice even if this procedure has a certain level of efficacy. As it requires a computed tomography-guided percutaneous approach, which is invasive, it has certain limitations in terms of methodology. In the case of pancreatic cancer, another study has shown the feasibility of endoscopic ultrasound-guided IRE ablation in an animal experiment. ${ }^{11}$ Therefore, we devised a method using endoscopic retrograde cholangiopancreatography (ERCP) as an alternative to insertion of an IRE ablation electrode into the bile duct without open surgery. First, an IRE ablation electrode in the form of a catheter, which can be inserted into the lesions in the bile duct after cannulation during ERCP, was developed. In this study, we conducted an experiment using an animal model to determine whether this method is effective and whether IRE treatment can be performed in the bile duct using ERCP.

\section{MATERIALS AND METHODS}

\section{Animals}

This study was conducted in three Yorkshire pigs (Orient Laboratory Animal Company, Seongnam, Korea) weighing $35 \pm 5 \mathrm{~kg}$. All pigs were housed for acclimation in an animal facility for 7 days. They were kept in the facility in 12-hr night and day intervals in a controlled room where constant temperature $\left(22^{\circ} \mathrm{C}-24^{\circ} \mathrm{C}\right)$ and humidity $(55 \%)$ were maintained. The animals were fasted the night before the experiment. After the intravenous injections of midazolam $0.5 \mathrm{~mL} / \mathrm{kg}$ and ketamine $25 \mathrm{mg} / \mathrm{kg}$, the procedure was performed under general anesthesia. This study was approved by the Institutional Animal Care \& Use Committee of Korea University (approval no. KOREA-2019-0146).

\section{Device}

The experiment was conducted using the newly designed
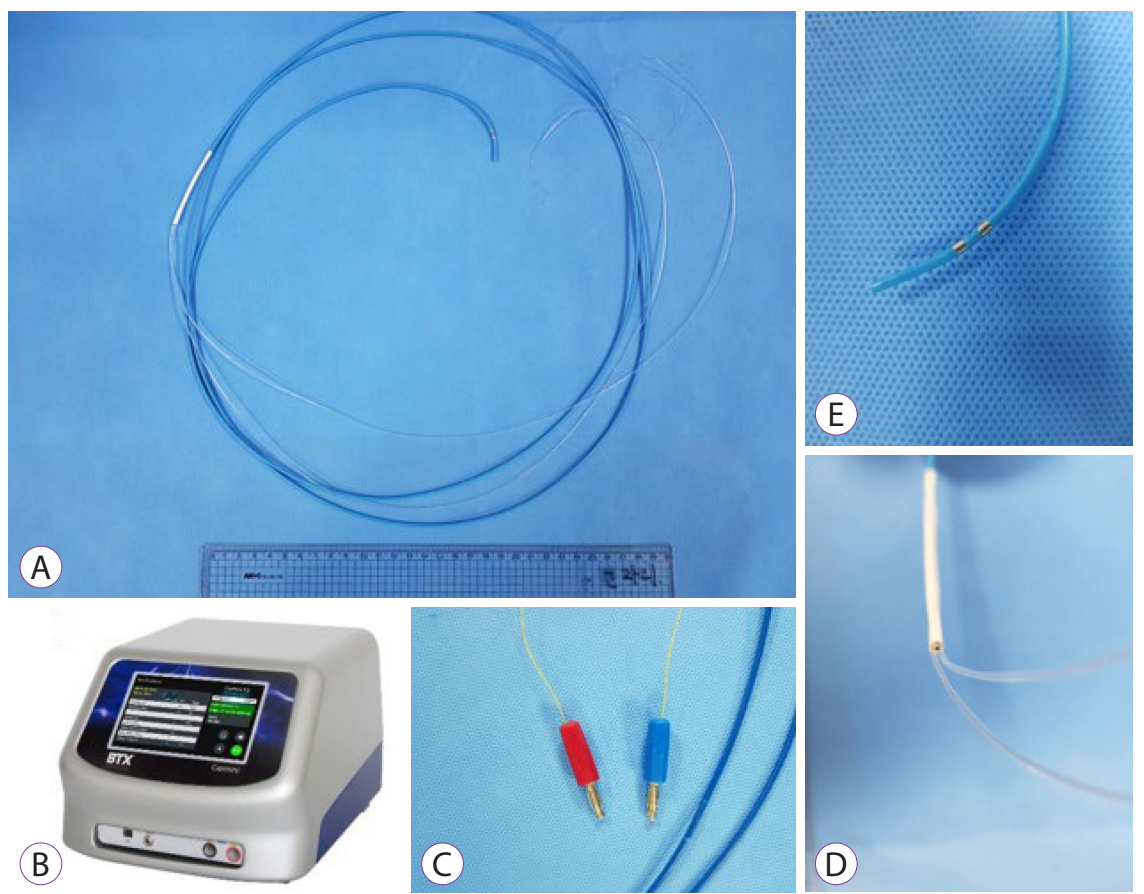

Fig. 1. Components of the newly designed endoscopic catheter-type irreversible electroporation electrode. Catheter-type irreversible electroporation (A) connecting to the main generator system. The Gemini Twin Wave BTX electroporation system (BTX Genetronics, San Diego, CA, USA) (B) consists of the banana connector $(C)$ along with insulated wires (D) and two electrodes arranged at 5 -mm intervals on the tip $(E)$ transmitting energy to the tissue. 
endoscopic catheter-type electrode. The device consisted of two electrodes (anode and cathode) arranged at 5-mm intervals on the tip and a long flexible catheter (shaped like an endoscopic nasobiliary drainage catheter) that can be inserted through the instrument channel of the endoscope. The cross section of the catheter is the structure in which the center hole through the guidewire and two insulated wires lead to the banana connector. The Gemini Twin Wave BTX electroporation system (BTX Genetronics, San Diego, CA, USA) was used for IRE ablation of the bile duct (Fig. 1). ERCP-guided IRE was performed using a duodenoscope (TJF-260V; Olympus, Tokyo, Japan).

\section{Procedure}

In this study, the catheter-shaped electrode was inserted into the bile duct during ERCP. The ERCP-guided approach was performed by placing the duodenoscope on the duodenum and subsequently inserting the guidewire into the bile duct after cannulation. After inserting the guidewire into the center hole of the electrode, the electrode was inserted through the instrument channel of the scope (Fig. 2). Through the C-arm $\mathrm{X}$-ray view, the electrode inserted along the guidewire was confirmed to be at a suitable location in the bile duct. The IRE protocol was proceeded following a setup that was considered to be appropriate in a previous pilot study. ${ }^{12}$ The energy was delivered in a pulse wave form in 10 sequences of 5 pulses, with a pulse duration of $100 \mu \mathrm{s}$, a pulse interval of $100 \mathrm{~ms}$, an amplitude of $500 \mathrm{~V} / \mathrm{cm}$, and a repetition frequency of $1 \mathrm{~Hz}$, with a 2-sec pause between the 5-pulse sequences.

Recent studies about the therapeutic effects of IRE for human or animal subjects were often conducted with a setting of 1,500-2,000 V/cm energy level. However, according to references, initial studies to check the safety of IRE began with a setting of 500-600 V/cm, which is the minimum energy level for IRE. ${ }^{6,13,14}$ As this study was an animal experiment with the aim of investigating the feasibility of a method of inserting the IRE probe into bile duct through ERCP, not through a percutaneous approach, the experiment was conducted with the minimum IRE energy of $500 \mathrm{~V} / \mathrm{cm}$ rather than high energy setting of $\geq 1,000 \mathrm{~V} / \mathrm{cm}$.

\section{Postprocedure assessment}

The pigs were sacrificed $24 \mathrm{hr}$ after the IRE procedure. The bile ducts of all sacrificed pigs were resected for histopathologic evaluation. First, the samples were visually checked and subsequently formalin fixed. The bile duct resected in the axial

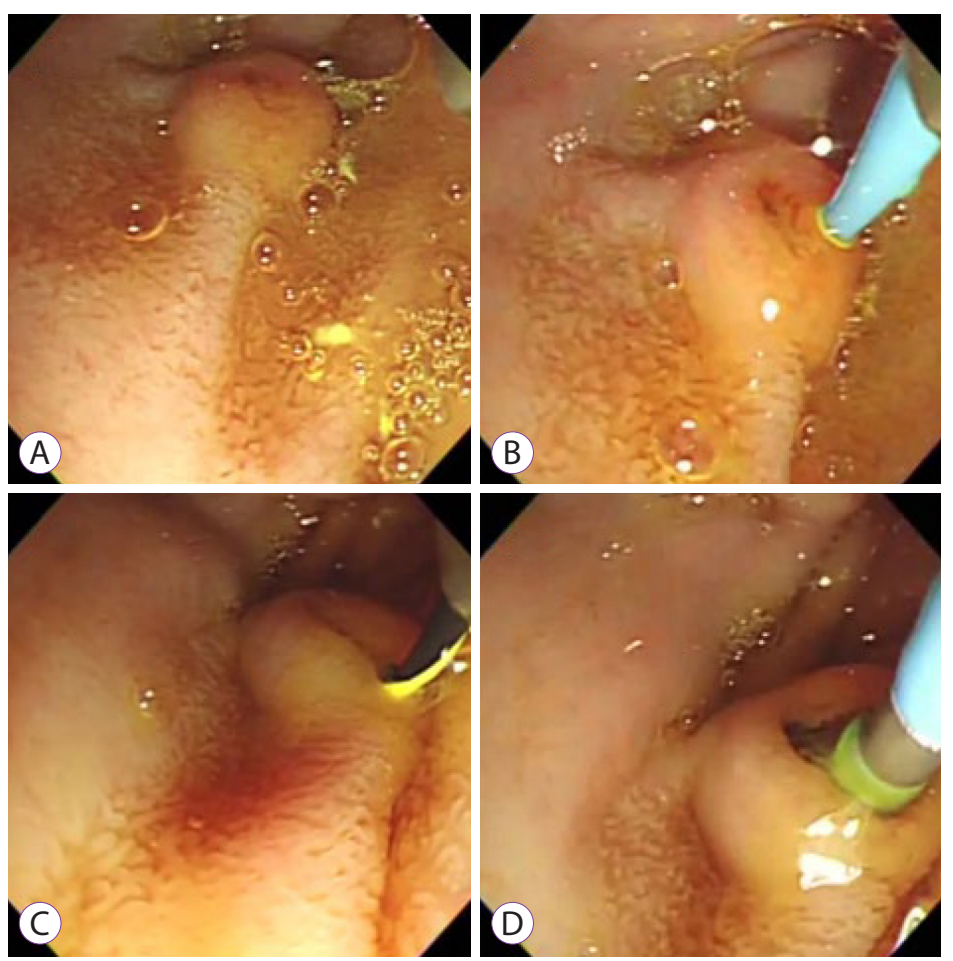

Fig. 2. Process of endoscopic retrograde cholangiopancreatography-guided endoscopic irreversible electroporation ablation. (A) After inserting the duodenoscope and targeting the ampulla of Vater, (B) cannulation was performed. (C) The guidewire was inserted, and (D) the electrode was inserted through the guidewire. 
plane, near the IRE application point, was sectioned again in the axial plane at 2-3 mm thickness intervals for tissue slides. Paraffin-embedded tissue slides were stained with hematoxylin and eosin (H\&E). Additionally, a terminal deoxynucleotidyl transferase-mediated deoxyuridine triphosphate digoxigenin nick end labeling (TUNEL) assay was performed. A digital slide was created using a slide scanner (Leica SCN400 Image Viewer; Leica, Wetzlar, Germany), and a precise analysis was performed using its viewer software.

\section{RESULTS}

\section{Ablation and clinical course}

The bile ducts of the three female Yorkshire pigs were examined. All three pigs underwent IRE ablation using the ERCP-guided approach. Under ERCP guidance, the IRE catheter was well visualized on an X-ray view with the band of the electrode. The process of inserting the catheter-shaped electrode through ERCP also did not significantly differ from that of a typical ERCP procedure. IRE ablation targeting the bile duct was technically successful. No technical problems, such as an electric short circuit, occurred during the IRE ablation of the bile duct. The IRE ablation, performed using an amplitude of $1,000 \mathrm{~V} / \mathrm{cm}$, was completed in three pigs. The procedure was completed in all pigs under general anesthesia. The pigs were observed for $24 \mathrm{hr}$ after the procedure, and no complications were noted.

\section{Histopathology}

The pigs were euthanized $24 \mathrm{hr}$ after the experiment. Thereafter, the bile ducts were resected and the luminal mucous membranes of the specimens were visually checked. The size of the ablated region in specimen $\# 1$ was $10 \times 5 \mathrm{~mm}$ and the depth was $3.1 \mathrm{~mm}$ (Fig. 3). The size and depth of the ablated region in specimen $\# 2$ and $\# 3$ were $13 \times 6 \mathrm{~mm}$ and $3.3 \mathrm{~mm}$ and $8 \times 5 \mathrm{~mm}$ and $2.6 \mathrm{~mm}$, respectively. The average length and width were $10.3 \times 5.3 \mathrm{~mm}$, and the average depth was 3 $\mathrm{mm}$ (Table 1). Well-demarcated focal color changes were observed on the mucosa of the bile duct where IRE was applied. The boundary of the ablated region was also visually apparent, as seen after formalin fixation (Fig. 4).

At a low magnification of the H\&E-stained slides, well-demarcated cellular changes were observed in the epithelium and lamina propria layer of the internal luminal wall of the bile duct. In the TUNEL assay, in which the tissue was dyed brown to detect apoptosis, apoptotic change was observed in the direction of the long axis of the bile duct. TUNEL immunohistochemistry showed cell death in the bile duct mucosa and submucosa along the electrode (Fig. 5). A positive TUNEL assay suggests a cellular change resulting from apoptosis rather than necrosis.

At higher magnifications, the IRE-ablated region showed loss of nucleus and fibrotic changes compared with the normal epithelium (Fig. 6). However, the ablation depth was within 3 $\mathrm{mm}$ from the lumen, which means that it barely reached the mucous membrane and the submucosal layer. This finding indicates that the ablation depth was still insufficient.

Histopathology revealed loss of epithelium and fibrotic change in the IRE-ablated area, as well as increased lymphocyte numbers and inflammatory cell infiltration around the ablated zone. The presence of edema of the duct wall was not clearly identified.
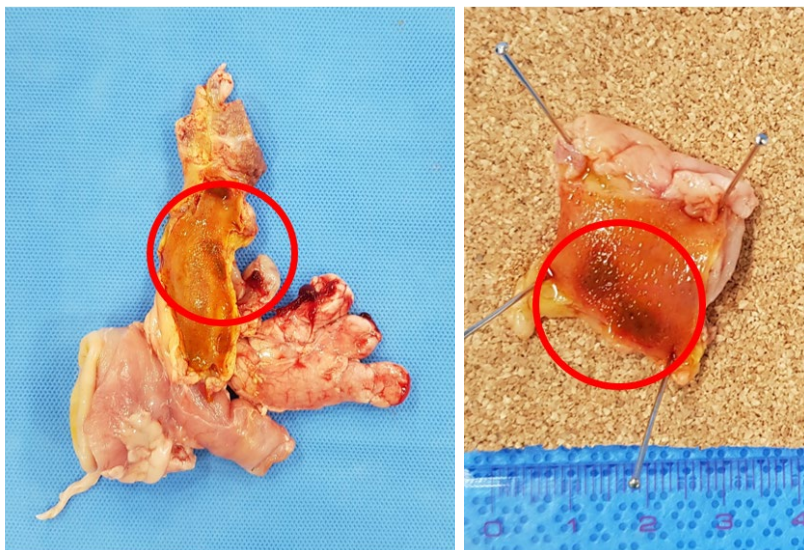

Fig. 3. Gross specimen of the bile duct after endoscopic retrograde cholangiopancreatography-guided endoscopic irreversible electroporation (IRE). In the red circle, the ablated region in the bile duct was $1 \mathrm{~cm}$ long and $5 \mathrm{~mm}$ wide. Well-demarcated focal color changes were observed on the mucosa of the bile duct where IRE was applied. No evidence of burn, bleeding, or any mucosal damage was observed in areas where IRE ablation was not applied.

Table 1. The Size of Ablated Site in Each Animal's Bile Duct Specimen

\begin{tabular}{lccc}
\hline Specimen & Length $(\mathbf{m m})^{\text {a) }}$ & Width $(\mathbf{m m})^{\text {a) }}$ & Depth $(\mathbf{m m})^{\text {b) }}$ \\
\hline Specimen \#1 & 10 & 5 & 3.1 \\
Specimen \#2 & 13 & 6 & 3.3 \\
Specimen \#3 & 8 & 5 & 2.6 \\
Average & 10.3 & 5.3 & 3.0 \\
\hline
\end{tabular}

Values are presented as number.

a) The visual findings of specimen were measured with a ruler. The result was round from the first decimal place.

b) The deepest depth was measured using a microscope ruler. The number is rounded to the second decimal place. 

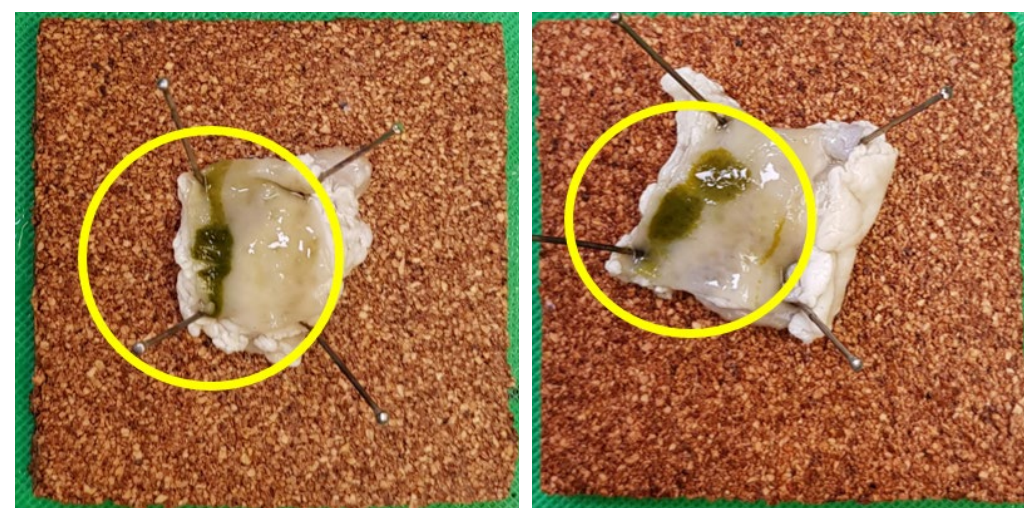

Fig. 4. Specimen with formalin fixation. In the yellow circle, the boundary of the ablated region was also visually apparent, as seen after formalin fixation.

H\&E stain
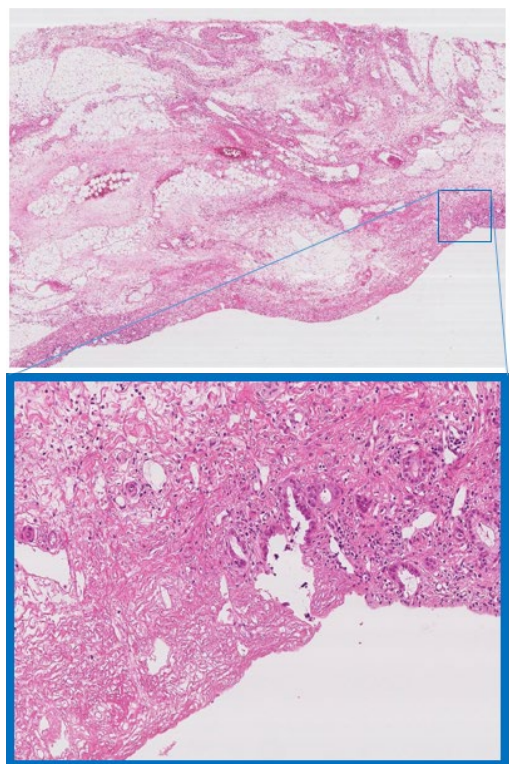

TUNEL assay
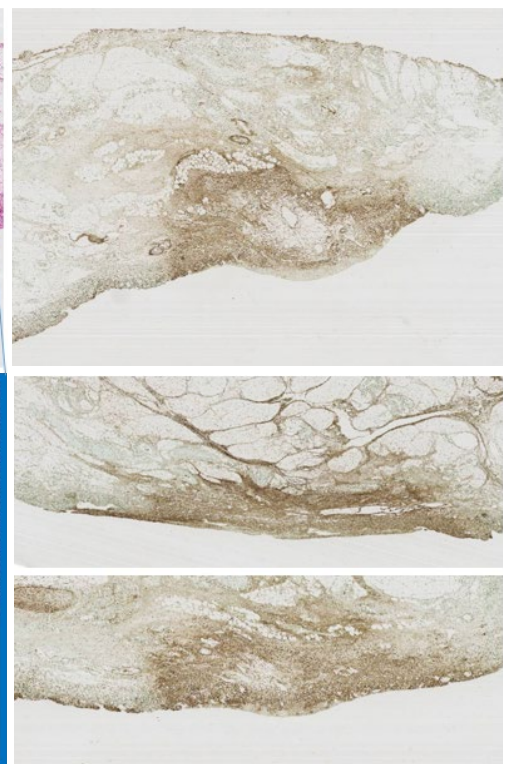

Fig. 5. Histopathologic findings of the bile duct in hematoxylin and eosin (H\&E) staining and terminal deoxynucleotidyl transferase-mediated deoxyuridine triphosphate digoxigenin nick end labeling (TUNEL) assay $(\times 40, \times 100)$. In the areas where irreversible electroporation was applied, the nucleus inside the cells were not observed (left). The area where irreversible electroporation was applied could be seen as a dark brown area in immunohistopathology with the TUNEL assay (right).

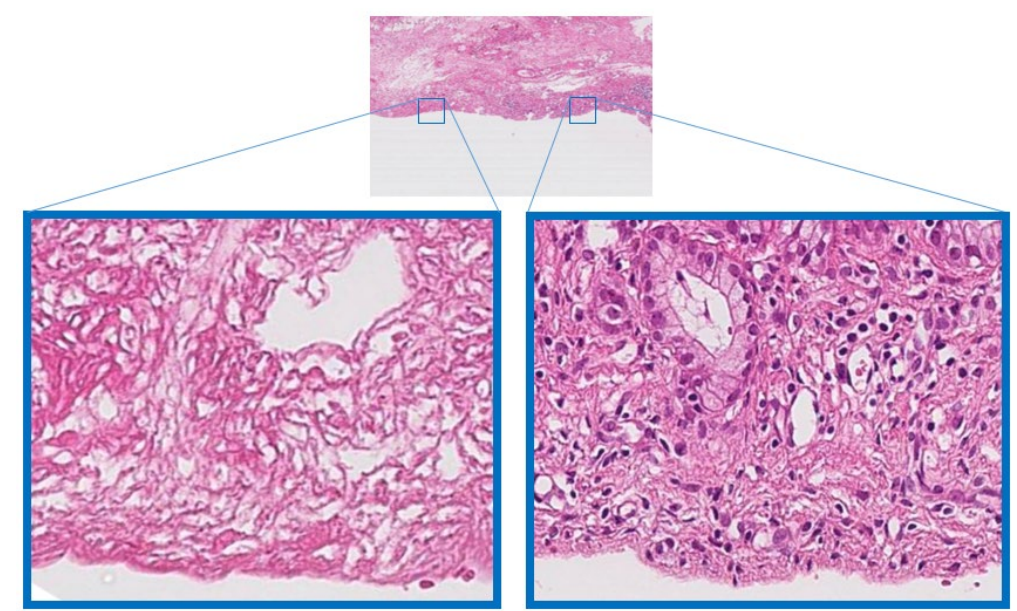

Fig. 6. Magnified images of the irreversible electroporation ablation area and the nonablated area in hematoxylin and eosin-stained sections $(\times 40, \times 400)$. Intracellular nuclei were observed in normal cells (right). However, the nuclei were not observed in the irreversible electroporation-ablated area (left). 


\section{DISCUSSION}

The current treatment based on systemic chemotherapy has limitations in improving the survival rate of patients with advanced cholangiocarcinoma. ${ }^{1}$ ERCP is an effective treatment option for bile duct stenosis, which is one of the major issues in patients with cholangiocarcinoma, through endoscopic decompression of the stenosis in unresectable cholangiocarcinoma. This procedure is frequently performed in clinical practice. However, it is difficult to expect an improvement in the survival rate with this endoscopic treatment. Moreover, this method should only be used as a palliative and supportive treatment. ${ }^{2}$ In addition to various studies using thermal or freezing methods such as radiofrequency ablation or cryotherapy, attempts of selective radioembolization using microspheres containing yttrium-90 have been made. However, these were only experimental trials owing to concerns about the possible occurrence of complications. ${ }^{15}$ Recent studies have been conducted to confirm whether IRE, a nonthermal method, could serve as a local tumor controller, rather than a thermal or freezing method with a high complication risk. ${ }^{16}$ Therefore, this study was planned with the expectation that IRE could be an effective treatment method if applied to the correct target lesion using a noninvasive approach via ERCP.

Cases of IRE performed in the pancreas, liver, prostate, and lungs have been reported in humans. Usman et al. reported an unsuccessful IRE ablation conducted using a computed tomography-guided percutaneous approach in two patients with unresectable lung tumors. ${ }^{17}$ The authors speculated that because the lung is not a solid organ, the air inside the lungs may have prohibited the deposition of energy. For this reason, IRE may have technical limitations when used as a local treatment for lung tumors. In previous human case studies, IRE ablation was effective for the treatment of tumors of the pancreas, liver, and prostate. A systematic review including four IRE studies conducted in patients with pancreatic cancer showed statistically significant differences in progression-free survival and overall survival between the IRE group and the non-IRE group. ${ }^{18}$ The review pointed out that a thermal procedure, such as radiofrequency ablation, for treating pancreatic cancer can damage the surrounding vascular structures; hence, scalability is limited. IRE does not cause a heat sink effect because it is a nonthermal-based ablation technique. Therefore, the structure and connective tissues of the target organ remain intact after the treatment. In Sutter et al., an IRE ablation targeting 75 hepatocellular carcinoma lesions in 58 patients showed an $87 \%$ and $70 \%$ progression-free survival at the 6-month and 12-month follow-up, respectively. ${ }^{19}$ Of 58 patients, 11 (19\%) developed complications, whereas 3 (5.2\%) had severe complications of grade III or higher. On the basis of this result, the authors argued that IRE ablation is a safe and effective treatment method for patients who are not suitable for conventional radiofrequency ablation. In a previous clinical study using IRE ablation for prostate cancer, although the treatment had negative effects on urinary and erectile function, cancer cells were identified in the ablated zone on a follow-up biopsy 6 months after treatment induction. ${ }^{20}$ Moreover, no residual cancer cells were noted in the ablated zone. Whether IRE ablation can improve the patients' survival is evident only when data are sufficient to confirm statistical significance. IRE ablation has received large attention from many researchers, as it is a promising option for cancer treatment.

Our experimental study was conducted to evaluate if ER$\mathrm{CP}$-guided IRE ablation with insertion of a catheter-type electrode is feasible, safe, and effective. We verified the feasibility of the procedure; that is, whether the catheter-shaped electrode could be inserted through the instrument channel of the duodenoscope without damaging the surrounding tissues. $\mathrm{C}$-arm X-ray confirmed that the electrode can be inserted into the target location in the bile duct of pigs. This technique has achieved technical advancement and can be directly applied to ERCP procedures in real clinical practice. It has also shown exceptional outcomes in terms of feasibility. After the IRE ablation, the pigs were closely monitored by experienced veterinarians every $8 \mathrm{hr}$ for $24 \mathrm{hr}$, and no critical complications were observed. Subsequent necropsy showed no evidence of perforation, peritonitis, or injury of vascular structures, similar to other studies conducted in different organs. ${ }^{21-23}$ Apoptosis, the result of electroporation, was confirmed by histopathologic evaluation; however, the evaluation showed insufficient depth of the ablated ductal wall. Compared with other studies, our study used lower energy levels as the wall of the bile duct with a tunnel-type structure is thinner than that of other solid organs such as the liver and pancreas. If the setting of IRE ablation is adjusted, it will cause sufficient apoptosis up to the target depth. On the basis of the results of this study, endoscopic IRE treatment using the newly developed catheter-type electrode is feasible and safe.

However, some limitations have been reported. First, the catheter-type electrode could not be used for ablation of the entire bile duct lumen. When ERCP-guided IRE ablation is used as a local treatment for bile duct tumors in the future, it is unclear if the electrodes can be evenly applied to an internal lumen narrowed by tumors. A solution is being considered to allow the electrodes to bond to the ballooning area of the catheter so that it can be applied evenly to the internal lumen of the bile duct. Another concern is the insufficient energy depth. To apply IRE ablation to cholangiocarcinoma lesions through ERCP in the future, setting to a low energy level for treatment should be considered a priority because of the narrow tunnel 
shape of the bile duct, the thin ductal wall, and the adjacent major vessels such as the portal vein. As confirmed by $\mathrm{H} \& \mathrm{E}$ staining and the TUNEL assay, significant cellular changes induced by IRE ablation were only observed 2-3 $\mathrm{mm}$ from the lumen and were limited to the mucosal and submucosal layers. To apply this technique to real-world practice, the ablation depth should be $>3 \mathrm{~mm}$ to induce transmural fibrosis and apoptosis. However, additional experiments are needed, taking into consideration the safety of the procedure at a higher energy intensity applied to reach the deeper layers. The IRE intensity used in this experiment did not cause any complications, such as burns. However, necrosis is expected to occur instead of apoptosis if higher energy intensities are used. ${ }^{24}$

Endoscopic IRE ablation using ERCP was successfully performed in the common bile duct of pigs using a catheter-shaped electrode. If sufficient data can be collected to allow statistical analysis for optimization of the applied parameters, IRE can be a potential minimally invasive ablation technique for the treatment of biliary tumors. Further efforts are needed to improve the current technique of ERCP-guided IRE ablation for the treatment of bile duct tumors.

Conflicts of Interest

The authors have no potential conflicts of interest.

Funding

This research was supported by a 2018 Weolbong grant from the Korean Gastrointestinal Endoscopy Research Foundation and by a National Research Foundation of Korea grant funded by the Korean government (no. 2018R1C1B6006044).

ORCID
Kang Won Lee:
Jae Min Lee:
Hyuk Soon Choi:
Eun Sun Kim:
Bora Keum:
Yeon Seok Seo:
Yoon Tae Jeen:
Soon Ho Um:
Hong Sik Lee:
Hoon Jai Chun:
Chang Duck Kim:
Chi Hyuk Oh:
Hong Bae Kim:

https://orcid.org/0000-0002-5902-9700 https://orcid.org/0000-0001-9553-5101 https://orcid.org/0000-0002-4343-6950 https://orcid.org/0000-0003-1820-459X https://orcid.org/0000-0003-0391-1945 https://orcid.org/0000-0003-4171-6331 https://orcid.org/0000-0003-0220-3816 https://orcid.org/0000-0002-4545-7907 https://orcid.org/0000-0001-9726-5416 https://orcid.org/0000-0002-5539-361X https://orcid.org/0000-0003-1027-7890 https://orcid.org/0000-0002-4382-5876 https://orcid.org/0000-0002-1661-6585

\section{REFERENCES}

1. Murakami Y, Uemura K, Sudo T, et al. Prognostic factors after surgical resection for intrahepatic, hilar, and distal cholangiocarcinoma. Ann Surg Oncol 2011;18:651-658.

2. Koch C, Franzke C, Bechstein WO, et al. Poor prognosis of advanced cholangiocarcinoma: real-world data from a tertiary referral center. Di- gestion 2020;101:458-465.

3. Aljiffry M, Walsh MJ, Molinari M. Advances in diagnosis, treatment and palliation of cholangiocarcinoma: 1990-2009. World J Gastroenterol 2009;15:4240-4262.

4. Wagstaff PG, Buijs M, van den Bos W, et al. Irreversible electroporation: state of the art. Onco Targets Ther 2016;9:2437-2446.

5. Curley SA, Marra P, Beaty K, et al. Early and late complications after radiofrequency ablation of malignant liver tumors in 608 patients. Ann Surg 2004;239:450-458.

6. Rubinsky B, Onik G, Mikus P. Irreversible electroporation: a new ablation modality--clinical implications. Technol Cancer Res Treat 2007;6:37-48.

7. Weaver JC, Chizmadzhev YA. Theory of electroporation: a review. Bioelectrochem Bioenerg 1996;41:135-160.

8. Miller L, Leor J, Rubinsky B. Cancer cells ablation with irreversible electroporation. Technol Cancer Res Treat 2005;4:699-705.

9. Belfiore MP, Ronza FM, Romano F, et al. Percutaneous CT-guided irreversible electroporation followed by chemotherapy as a novel neoadjuvant protocol in locally advanced pancreatic cancer: our preliminary experience. Int J Surg 2015;21(Suppl 1):S34-S39.

10. Hsiao CY, Huang KW. Irreversible electroporation: a novel ultrasound-guided modality for non-thermal tumor ablation. J Med Ultrasound 2017;25:195-200.

11. Lee JM, Choi HS, Chun HJ, et al. EUS-guided irreversible electroporation using endoscopic needle-electrode in porcine pancreas. Surg Endosc 2019;33:658-662.

12. Lee JM, Choi HS, Kim ES, et al. Characterization of irreversible electroporation on the stomach: a feasibility study in rats. Sci Rep 2019;9:9094.

13. Davalos RV, Mir IL, Rubinsky B. Tissue ablation with irreversible electroporation. Ann Biomed Eng 2005;33:223-231.

14. Edd JF, Horowitz L, Davalos RV, Mir LM, Rubinsky B. In vivo results of a new focal tissue ablation technique: irreversible electroporation. IEEE Trans Biomed Eng 2006;53:1409-1415.

15. Jung CFM, Lavole J, Barret M, et al. Local therapy in advanced cholangiocarcinoma: a review of current endoscopic, medical, and oncologic treatment options. Oncology 2019;97:191-201.

16. Scheffer HJ, Nielsen K, de Jong MC, et al. Irreversible electroporation for nonthermal tumor ablation in the clinical setting: a systematic review of safety and efficacy. J Vasc Interv Radiol 2014;25:997-1011; quiz 1011.

17. Usman M, Moore W, Talati R, Watkins K, Bilfinger TV. Irreversible electroporation of lung neoplasm: a case series. Med Sci Monit 2012;18:CS43-CS47.

18. Moir J, White SA, French JJ, Littler P, Manas DM. Systematic review of irreversible electroporation in the treatment of advanced pancreatic cancer. Eur J Surg Oncol 2014;40:1598-1604.

19. Sutter O, Calvo J, N'Kontchou G, et al. Safety and efficacy of irreversible electroporation for the treatment of hepatocellular carcinoma not amenable to thermal ablation techniques: a retrospective single-center case series. Radiology 2017;284:877-886.

20. Murray KS, Ehdaie B, Musser J, et al. Pilot study to assess safety and clinical outcomes of irreversible electroporation for partial gland ablation in men with prostate cancer. J Urol 2016;196:883-890.

21. Luo X, Liang X, Li J, et al. The effects of irreversible electroporation on the colon in a porcine model. PLoS One 2016;11:e0167275.

22. Lee EW, Wong D, Tafti BA, et al. Irreversible electroporation in eradication of rabbit VX2 liver tumor. J Vasc Interv Radiol 2012;23:833-840.

23. Zhang W, Wang W, Chai W, et al. Breast tissue ablation with irreversible electroporation in rabbits: a safety and feasibility study. PLoS One 2017;12:e0181555.

24. Cvetković DM, Živanović MN, Milutinović MG, et al. Real-time monitoring of cytotoxic effects of electroporation on breast and colon cancer cell lines. Bioelectrochemistry 2017;113:85-94. 